\title{
Exploring water and other liquids at negative pressure
}

\author{
Frédéric Caupin ${ }^{1,2}$, Arnaud Arvengas ${ }^{1}$, Kristina Davitt ${ }^{1}$, Mouna El Mekki Azouzi ${ }^{2,3}$, Kirill I Shmulovich ${ }^{4}$, \\ Claire Ramboz ${ }^{3}$, David A Sessoms ${ }^{5}$ and Abraham D Stroock ${ }^{5}$
}

\begin{abstract}
Water is famous for its anomalies, most of which become dramatic in the supercooled region, where the liquid is metastable with respect to the solid. Another metastable region has been hitherto less studied: the region where the pressure is negative. Here we review the work on the liquid in the stretched state. Characterization of the properties of the metastable liquid before it breaks by nucleation of a vapour bubble (cavitation) is a challenging task. The recent measurement of the equation of state of the liquid at room temperature down to $-26 \mathrm{MPa}$ opens the way to more detailed information on water at low density. The threshold for cavitation in stretched water has also been studied by several methods. A puzzling discrepancy between experiments and theory remains unexplained. To evaluate how specific this behaviour is to water, we discuss the cavitation data on other liquids. We conclude with a description of the ongoing work in our groups.
\end{abstract}

\section{Introduction to metastability and negative pressures}

\subsection{Metastability and nucleation}

First-order transitions are the most familiar transitions between phases of the same substance. Two phases, e.g. liquid and solid or liquid and vapour, can be observed in equilibrium along a coexistence line in the phase diagram. Away from this line, only one of the phases is stable. Yet, the other one might still be observed: it is then called metastable. In the energy landscape connecting the two phases, there is a saddle point which defines the energy barrier $E_{\mathrm{b}}$ that has to be overcome for the transformation to occur. The jump over the barrier will occur because of fluctuations in the metastable system, with a rate per unit volume and time:

$\Gamma=\Gamma_{0} \exp \left(-\frac{E_{\mathrm{b}}}{k_{\mathrm{B}} T}\right)$

where $k_{\mathrm{B}}$ is the Boltzmann constant, $T$ the temperature and $\Gamma_{0}$ a prefactor whose expression varies [1]. Anyhow, because of the exponential, the exact value of $\Gamma_{0}$ has much less importance than the barrier $E_{\mathrm{b}}$. If the barrier is large, the rate will be small, and a significant volume of the metastable phase may be observed during a long time, allowing experiments to be performed on it.

Knowledge of the barrier is therefore required to predict the range of metastability accessible to experiments. The simplest approach is provided by classical nucleation theory (CNT). It assumes that the transition occurs through the growth of a spherical bubble of the stable phase inside the metastable one and uses the macroscopic interfacial tension between the two phases to calculate the energy barrier. A detailed discussion of CNT can be found in [1]. For 
illustration, we give here the minimum work required to create a spherical bubble of vapour with a radius $R$ in a liquid at pressure $P$ :

$W(R)=\frac{4}{3} \pi R^{3}\left(P-P_{\text {sat }}\right)+4 \pi R^{2} \sigma_{\mathrm{LV}}$,

where $\sigma_{\mathrm{LV}}$ is the bulk liquid-vapour interfacial tension. This is valid at sufficiently low temperatures; corrections apply near the critical point [1]. $W(R)$ reaches a maximum at the critical radius:

$R_{\mathrm{c}}=\frac{2 \sigma_{\mathrm{LV}}}{P_{\mathrm{sat}}-P}$

This expression, identical to Laplace's law, shows that the critical bubble is in unstable mechanical equilibrium: bubbles with radii smaller than $R_{\mathrm{c}}$ will decay, whereas bubbles with radii larger than $R_{\mathrm{c}}$ will grow. Finally, one gets the energy barrier

$E_{\mathrm{b}}=W\left(R_{\mathrm{c}}\right)=\frac{16 \pi}{3} \frac{\sigma_{\mathrm{LV}}^{3}}{\left(P_{\mathrm{sat}}-P\right)^{2}}$.

The liquid will reach its limit of metastability when the energy barrier becomes low enough to be overcome by thermal fluctuations. More specifically, consider an experiment on a volume $V_{\text {exp }}$ during a time $\tau_{\text {exp }}$; the probability $\Sigma$ of nucleation of a bubble is

$\Sigma=1-\exp \left[-\Gamma_{0} V_{\exp } \tau_{\exp } \exp \left(-\frac{E_{\mathrm{b}}}{k_{\mathrm{B}} T}\right)\right]$.

Defining the cavitation pressure as the pressure at which $\Sigma=1 / 2$, and using equation (4), one gets

$P_{\text {cav }}^{\mathrm{CNT}}=P_{\text {sat }}-\left[\frac{3 k_{\mathrm{B}} T}{16 \pi \sigma^{3}} \ln \left(\frac{\Gamma_{0} V \tau}{\ln 2}\right)\right]^{-1 / 2}$

with $\Gamma_{0} V \tau=10^{19}$, typical for acoustic cavitation in water [2], CNT predicts for water at room temperature a very negative cavitation pressure: $P_{\text {cav }}=-190 \mathrm{MPa}$.

\subsection{Why study negative pressures?}

Few experiments have been performed on liquids at negative pressure, because it is difficult to get rid of impurities or pre-existing bubbles that trigger cavitation. However, this exotic region of the phase diagram is interesting, because it allows us to probe directly the cohesion of matter: the molecules of the metastable liquid stick together because of their long range attractive interaction. 
This state is not a mere laboratory curiosity, it also exists in nature. The most famous example is the sap in trees. Indeed, negative pressures are needed for the sap to ascend a tree under certain conditions. The simplest way to see why negative pressures are necessary is to consider the law of hydrostatics. The pressure along a column of water of height $h$ decreases by $\rho g h$ from the bottom to the top. If the pressure at the roots is $0.1 \mathrm{MPa}$, the pressure above $10.2 \mathrm{~m}$ must therefore be negative. Reality is more complex, and other factors (drought, viscous flow, root pressure, etc) must be taken into account, but the picture most widely accepted today is the cohesion-tension theory where water from the soil is pulled up the tree thanks to a negative pressure at the top. For more details, see, for instance, [3] and [4].

Another example is capillary water. A narrow capillary filled with water that wets its walls exhibits a curved liquid-vapour interface. Because of Laplace's law, there is a pressure jump across the interface. Using the bulk surface tension of water $\sigma$, the jump $2 \sigma / R$ reaches $0.1 \mathrm{MPa}$ when the radius of curvature $R$ is smaller than $1.4 \mu \mathrm{m}$. Water in soil can thus be at negative pressure. This may also be relevant to the study of water confined in pores. Note, however, that for very narrow pores the pressure is not isotropic any more, the liquid/wall interaction introducing nondiagonal terms in the stress tensor.

\section{Water, a complex liquid}

Water is famous for its anomalies. Arguably the most cited is its line of density maxima, around $4{ }^{\circ} \mathrm{C}$ at atmospheric pressure. But many other thermodynamic and dynamic anomalies have been found and they become even stronger in the metastable region of the phase diagram where the liquid is supercooled with respect to the solid $[5,6]$. The large increase in many quantities has even suggested a power law divergence at a common temperature (see figure 1), which cannot be accessed experimentally because of the onset of crystallization. 


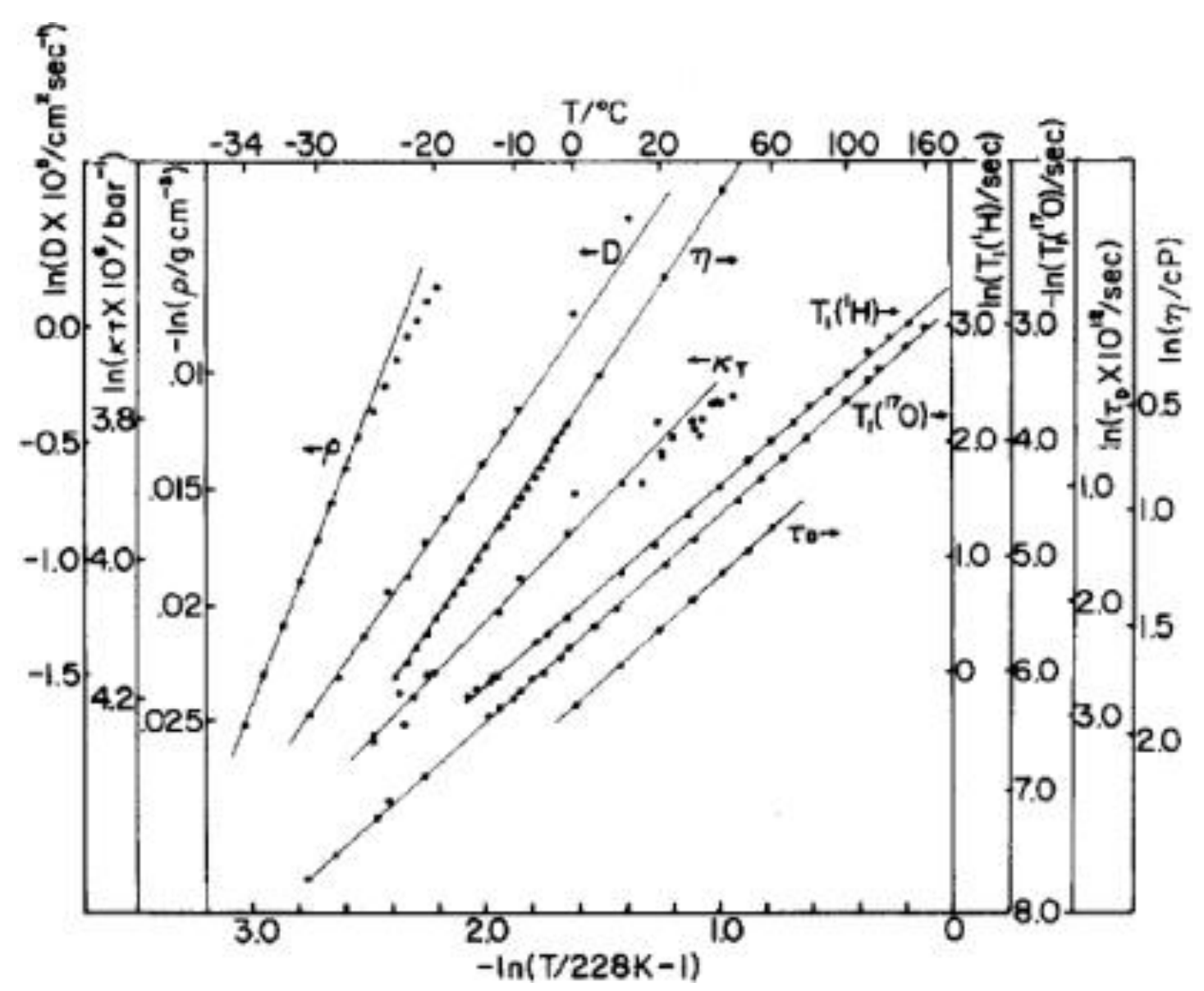

Figure 1. Compilation of some of the anomalies measured in supercooled water at atmospheric pressure plotted to illustrate their power law behaviour. Reprinted with permission from [5].

Several scenarios have been proposed to account for this behaviour. For a detailed discussion, we refer the reader to [1] and [6]. We would like to cite here only two scenarios, because they make the connection with the other metastable region where the liquid is stretched at negative pressure. In a first scenario due to Speedy [7], it is proposed that the divergence would be due to the re-entrance of the liquid-vapour spinodal. This is illustrated in figure 2. Speedy showed by thermodynamic arguments that, if the line of density maxima intersects the liquid-gas spinodal, it would have a minimum in the pressure-temperature plane. Several extrapolated equations of state (EoSs) based on data at positive pressure support the existence of this minimum. Even if the re-entrance up to positive pressure is a matter of debate $[6,8,9]$, the possibility of a nonmonotonic spinodal is interesting; up to now, it has been seen only in helium 3 [10]. 

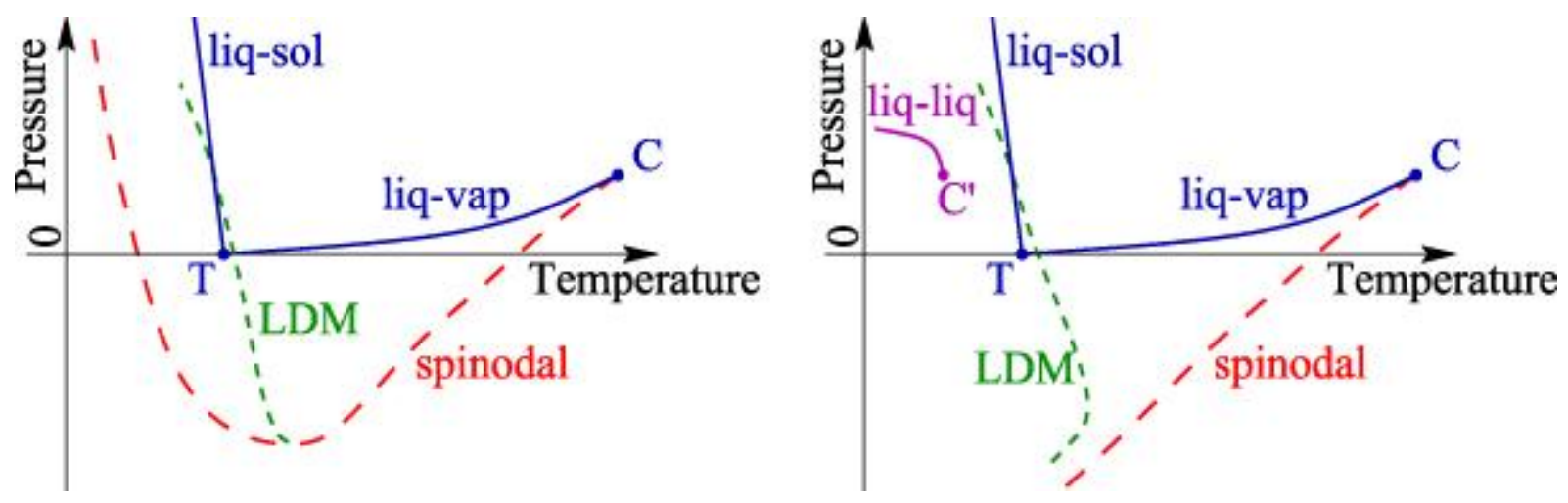

Figure 2. Two scenarios proposed to explain the anomalies of supercooled water. Left: retracing spinodal [7]. Right: liquid-liquid critical point [11]. The figures show the liquidvapour and liquid-solid equilibrium (solid blue lines), the line of density maxima (short dashed green line), the liquid-vapour spinodal (long dashed red line) and in the right panel the liquid-liquid transition (solid purple line).

Another scenario, originally proposed in [11], is based on molecular dynamics simulations. Using several water potentials, all simulations to date find that the line of density maxima does not intersect the liquid-gas spinodal, which remains monotonic (figure 2). The anomalies of supercooled water are then not due to the liquid-vapour spinodal. Instead, simulations find their origin in the vicinity of a critical point that would terminate a line of first-order transitions between two liquid phases. The two liquids, low and high density, would differ by the structure of the hydrogen bond network. However, experiments on bulk water have not been able to reach this liquid-liquid transition, because crystallization always happens first. Interestingly, a few simulations have found a liquid-liquid critical point at negative pressure [12-14]; however, they have not been confirmed by successive investigations $[15,16]$. In any case, the ideas put forward in the simulations are an additional motivation for the experimental study of stretched water.

\section{The equation of state of stretched water}

One of the differences between the scenarios presented above lies in the shape of the liquidgas spinodal line. Thus, it would be interesting to determine it experimentally. For this, measurement of the equation of state as far as possible in the metastable region at negative pressure is required. In this section, we describe the few experimental results available.

\subsection{The experiment by Meyer (1911)}

The first attempt to measure PVT data on stretched water dates back to Meyer, who published his results one hundred years ago [17]. To generate negative pressure, Meyer used a technique designed by Berthelot in 1850 [18]. A glass container is nearly filled with water and sealed hermetically, so that only a small bubble remains. Upon warming, the liquid expands and the bubble shrinks, until it eventually disappears. When the system is cooled down again, the liquid sticks to the walls and the bubble does not reappear. The liquid thus occupies a larger volume than during warming: it is stretched and a negative pressure builds up. At some point 
during cooling, the metastable liquid will break up by cavitation and a new bubble will be formed.

Meyer had the idea to modify the Berthelot tube by giving it the shape of a helix. When the pressure inside varies while the outside remains at atmospheric pressure, the helix coils or uncoils. This is the principle of the Bourdon gauge. Calibration by applying a positive pressure outside allows one to directly measure the pressure of the stretched liquid by measuring the angle of rotation of the helix. Meyer was able to simultaneously measure the volume of the liquid, thus obtaining a set of PVT data during cooling.

The largest tension he reached in water was $-3.4 \mathrm{MPa}$ at $24{ }^{\circ} \mathrm{C}$. Down to this point, he measured a linear pressure-volume relation for water. Note that he found a similar result for ethanol but detected a curvature in ether.

\subsection{Later work}

Another technique was extensively used by Briggs in 1950 [19], following a method first used by Reynolds. Negative pressure was generated by centrifugation. A capillary tube is attached to the axis of a centrifuge and filled with water. The tube ends are curved back to get an overall $\mathrm{Z}$ shape that prevents the water from spilling out when the tube is rotated. The centrifuge force creates a tension on the liquid, which is maximum on the axis:

$P_{\min }=P_{0}-\frac{1}{2} \rho \omega^{2} r^{2}$,

where $P_{0}$ is the pressure outside the tube, $\rho$ is the water density and $r$ is the distance between the centre and the liquid-air interface.

Briggs determined the cavitation pressure in several liquids. Winnick and Cho had the idea to measure the volume change of the liquid column as a function of angular velocity to obtain PVT data [20]. However, the analysis is complicated by the fact that the pressure in the liquid varies from $P_{\min }$ to atmospheric pressure. The published results, reaching $-10 \mathrm{MPa}$, were debated and no clear conclusion reached [21, 20, 22].

In the 1970s, Henderson and Speedy revisited the Meyer method based on Berthelot-Bourdon tubes [23]. Unfortunately, they were not able to measure the liquid volume accurately enough to obtain PVT data. Rather, they located the line of density maxima using the point at which a minimum pressure was reached when cooling the tube $[23,24]$. Indeed, when entering in the region where the expansion coefficient is negative, the tension in the tube is gradually released. They found that the line of density maxima extends linearly in the stretched region down to $-20.3 \mathrm{MPa}$.

\subsection{An isotherm down to $-26 \mathrm{MPa}$}

We have seen that only Meyer's experiment reported PVT data for stretched water. The limitation of his technique is that only a moderate tension of $-3 \mathrm{MPa}$ could be produced, presumably because of heterogeneous nucleation of vapour on the tube walls. There is a technique that is not subject to this limitation: acoustic cavitation. It uses an acoustic wave focused at the centre of a hemisphere. Large negative pressures, down to $-30 \mathrm{MPa}$, can thus be reached in a small region during a short time, and far away from any wall. This method has 
been used to measure the cavitation threshold in water [2]. To measure the EoS, Davitt et al chose to determine the thermodynamic parameters of the stretched liquid using optical methods. A fibre optic probe hydrophone was used to measure the density [25, 26]. The tip of a fibre is placed at the acoustic focus; the sound wave modulates the reflectivity of the liquid/fibre interface, which allows the refractive index to be measured. It is converted into density using an accurate phenomenological equation based on the Lorentz-Lorenz relation. The other parameter measured was the sound velocity of the stretched liquid, using Brillouin scattering [27]. When light interacts with thermal density fluctuations in the liquids, it can be scattered with a shift in frequency, proportional to the product of the refractive index and the sound velocity. However, in the acoustic experiment, the maximum negative pressure is reached only during a short time, typically $100 \mathrm{~ns}$. This difficulty was overcome by building a time-resolved Brillouin scattering experiment, using a gated avalanche photodiode and a large number of repetitions of the same sound bursts. Strictly speaking, the measurement in the sound wave is made along an isentrope, but in water at room temperature this is very close to an isotherm. The result is shown in figure 3. It is compared with the currently most accurate equation of state for water from the IAPWS [28, 29], based on experimental data at positive pressure. The extrapolation of this EOS is found to agree with the measurement by Davitt et al within the experimental error bars.

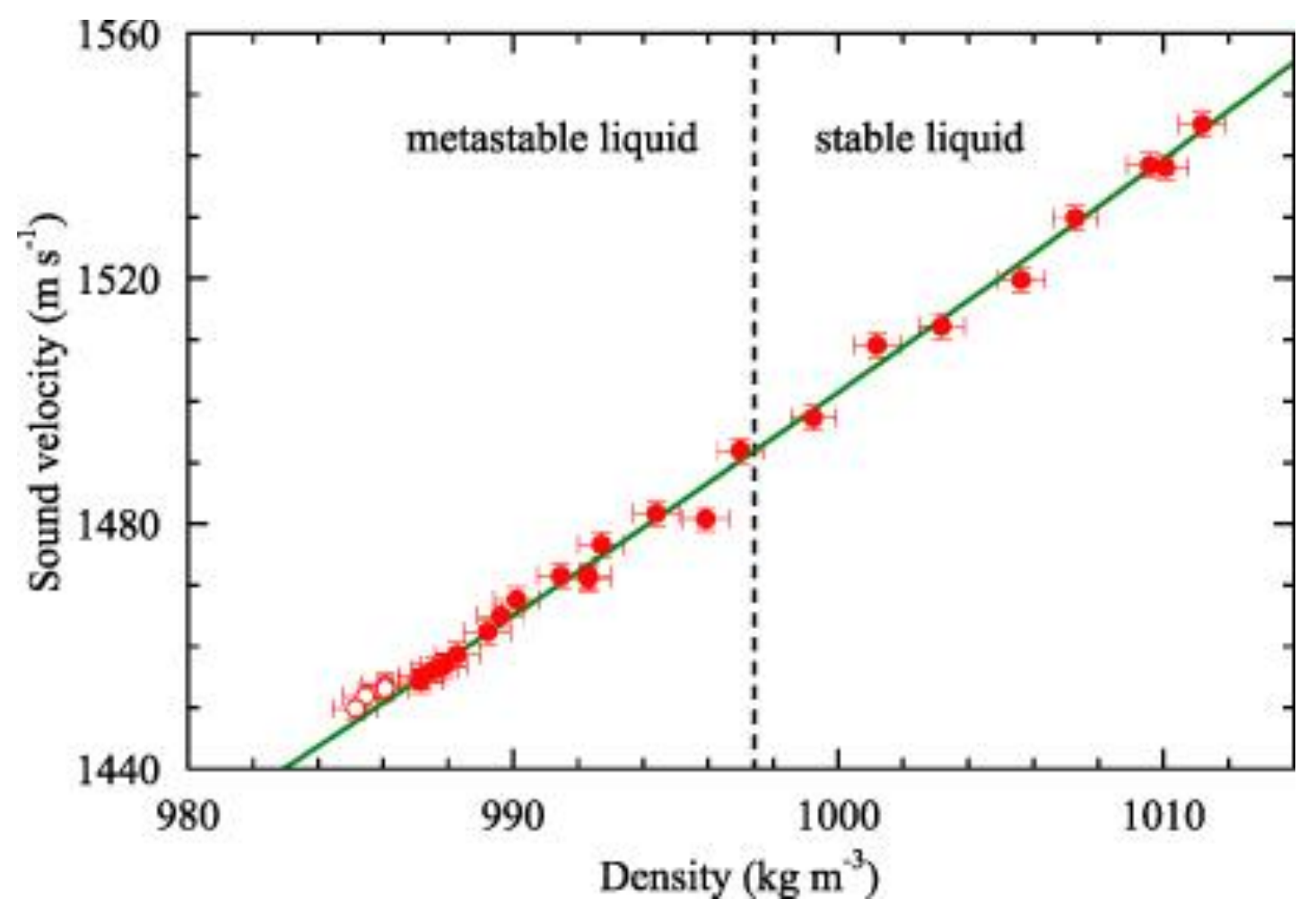

Figure 3. Sound velocity as a function of density in liquid water at $23.3^{\circ} \mathrm{C}$. The experimental data (red circles) is compared to the IAPWS EoS and its extrapolation (green solid curve). Adapted from [27].

\section{The enigma of the cavitation pressure of water}

We now turn to the cavitation pressure. In principle, it could also shed light on the debate about the different scenarios for water, because the temperature dependence of the cavitation pressure should reflect the behaviour (monotonic or not) of the spinodal line [30]. 
The experimental values are widely scattered, but in the cleanest experiments, values around $-30 \mathrm{MPa}$ are obtained. The most successful techniques are: centrifugation [19] and Berthelot tube [23, 24, 31] mentioned above, different acoustic techniques [32-34], including ours [35], and recently artificial trees [36]. The latter was developed at Cornell in the group of Stroock, mimicking the mechanism by which the leaves generate the negative pressure that pulls sap up a tree. It consists in equilibrating bulk water with undersaturated vapour at $P_{\text {vap }}<P_{\text {sat }}$ through a porous membrane, made with a poly(hydroxyethyl methacrylate) hydrogel. The chemical potentials of the liquid and the vapour phases become equal, so that

$$
\begin{aligned}
\mu_{\text {liq }}\left(T, P_{\text {liq }}\right)= & \mu_{\text {vap }}\left(T, P_{\text {vap }}\right) \\
& =\mu_{\text {vap }}\left(T, P_{\text {sat }}\right)+k_{\mathrm{B}} T \ln \frac{P_{\text {vap }}}{P_{\text {sat }}}
\end{aligned}
$$

where the vapour has been treated as a perfect gas. If the liquid is assumed to be incompressible with a volume per particle $v_{\text {liq }}$, this leads to

$P_{\text {liq }}=P_{\text {sat }}+\frac{k_{\mathrm{B}} T}{v_{\text {liq }}} \ln \frac{P_{\text {vap }}}{P_{\text {sat }}}$

which yields negative pressure for moderate undersaturation. For instance, $93 \%$ of relative humidity yields $P_{\text {liq }}=-10 \mathrm{MPa}$.

The thresholds obtained with these different techniques are compared on figure 4. A first comparison was given in [37]. In the present plot, we have added the data from the artificial trees [36]. We have also modified the data for our acoustic experiment: the previous plot used an indirect estimate of the cavitation pressure [2]; here we have used the extrapolation of the IAPWS EoS, which is supported by the results of Davitt et al to convert the cavitation density measured with a fibre optic probe hydrophone [35] into pressure. All the cavitation thresholds fall in the same range, our acoustic data being the most negative. Based on our data, the minimum in the cavitation pressure found by Briggs [19] seems to be an artefact. Despite the proximity of all these results, they are very far from the theoretical expectations (e.g. around $-190 \mathrm{MPa}$ at room temperature based on CNT, see section 1.1). Therefore, the controversy about the spinodal limit cannot be settled with this data. 


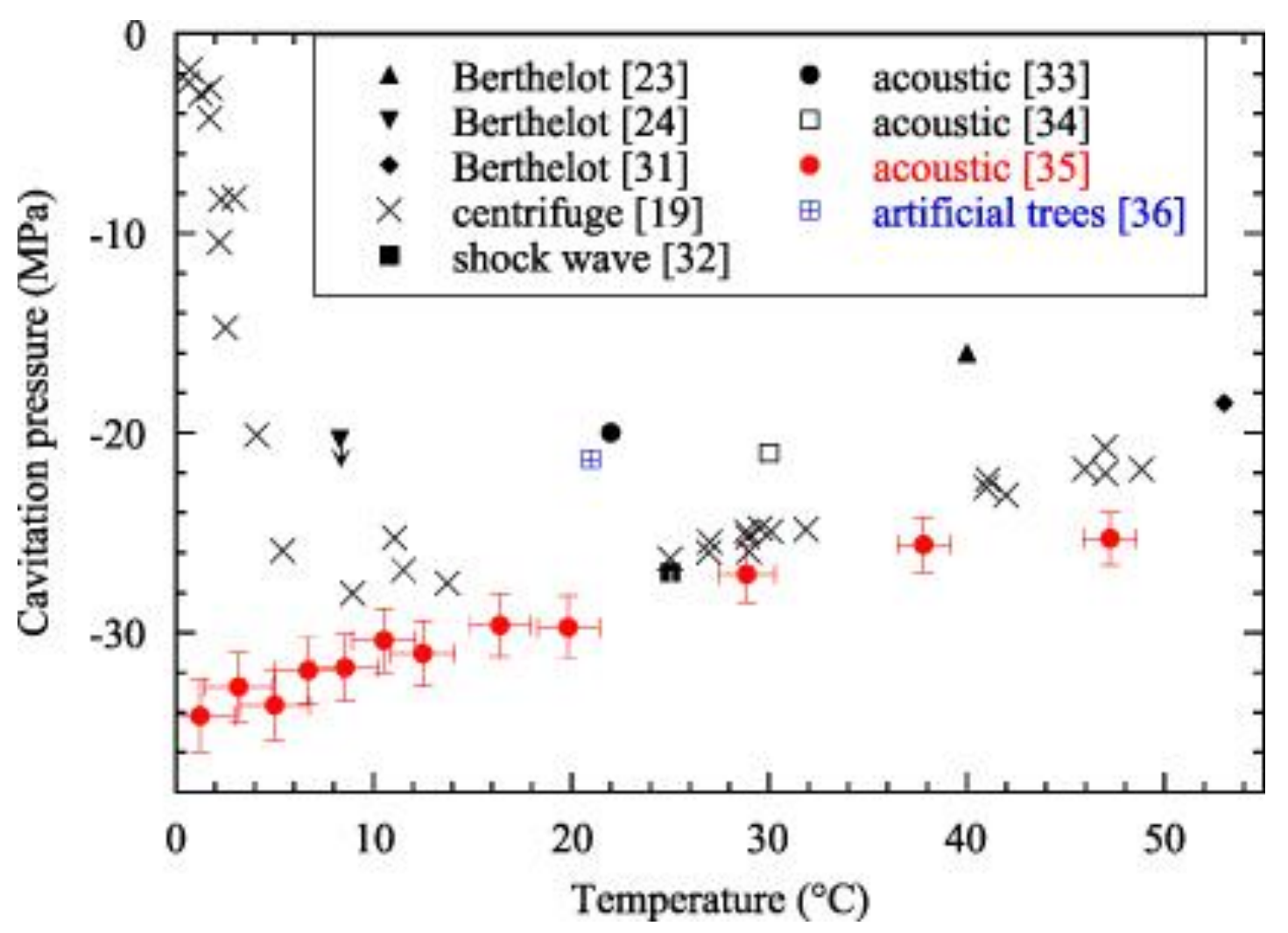

Figure 4. Cavitation pressure as a function of temperature for different experiments: the corresponding method and reference are given in the legend. An arrow means that cavitation was not observed. The full circles represent the pressure converted with IAPWS EoS from the density measurements using the FOPH [26]. The blue crossed square is the result using artificial trees, with the pressure calculated from the controlled vapour activity [36].

However, there is one experimental technique that is sometimes able to reach cavitation pressures close to theory. It uses the Berthelot tube technique described in section 3.1, but this time the container is a microscopic cavity in a quartz crystal, in the $10 \mu \mathrm{m}$ range. Using these quartz inclusions, the group of Angell has reached a largest negative pressure estimated to be $-140 \mathrm{MPa}$ [38]. The estimate was based on an extrapolated EoS, using the density obtained from the temperature at which the bubble in the Berthelot tube disappears upon heating, and assuming that the inclusion volume does not change with temperature. A Brillouin scattering study [39] showed that this assumption could be wrong in very thin inclusions, but that it seemed correct in more rounded ones.

The quartz inclusion technique was recently reproduced in Orléans, yielding similar results [40]. Several aqueous solutions were also studied. Figure 5 displays a new set of pure water inclusions studied by El Mekki, which confirms the published results. The measurements with one given inclusion are reproducible. However, for different inclusions, even within the same quartz sample, the results are very scattered. In contrast, the recent acoustic experiment exhibits extreme reproducibility. However, the largest negative pressure reached in the quartz inclusions is much closer to the prediction of CNT. Note also that the data scattering was strongly reduced if, instead of pure water, a better solvent for the quartz 
crystal (sodium hydroxide solution) was used, which improved the quality of the surfaces of the inclusions [40].

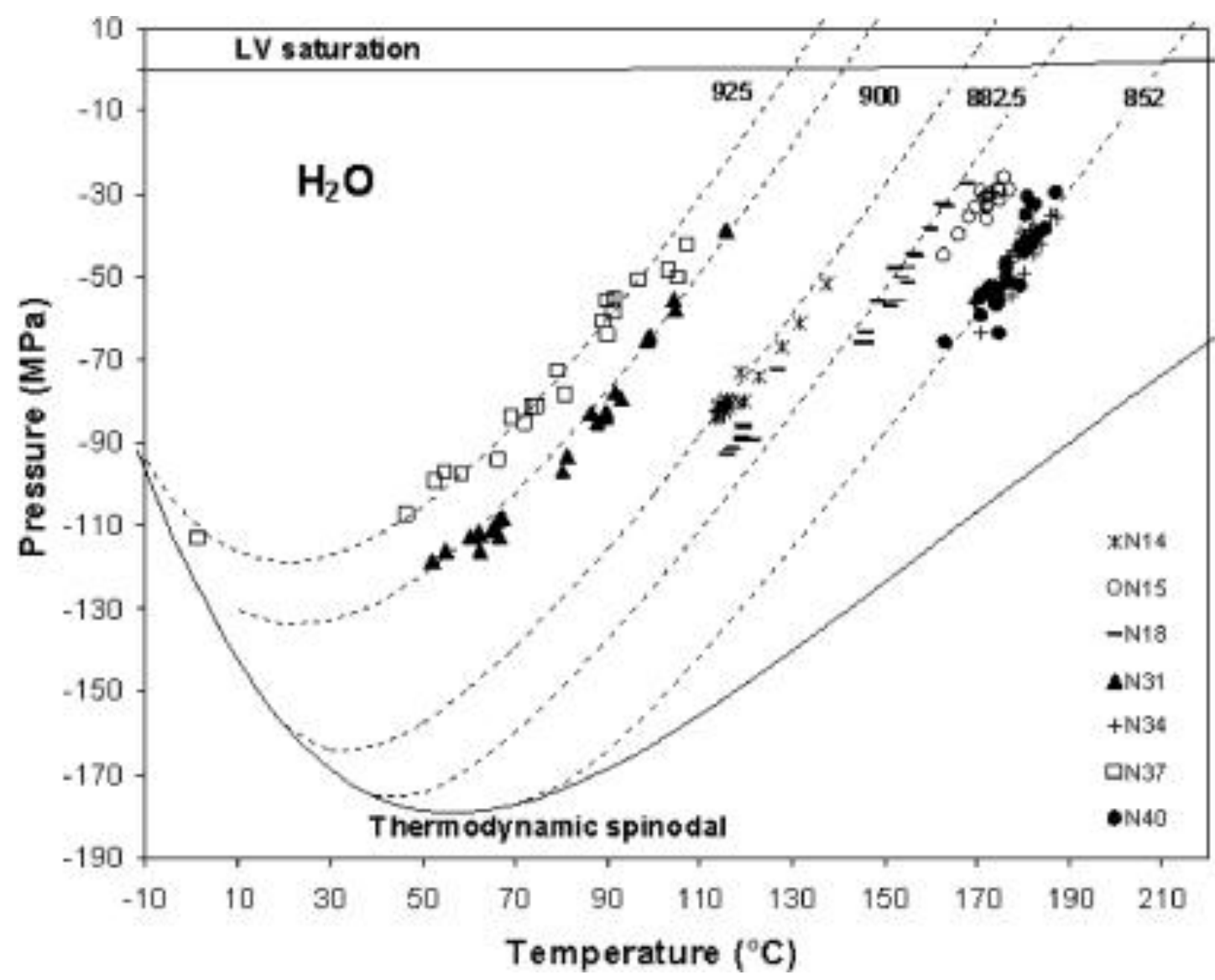

Figure 5. Cavitation pressure as a function of temperature for water inclusions in quartz. The data are unpublished results from the samples studied in [40]. The isochores and the spinodal were obtained using the extrapolation of the IAPWS EoS.

The isochoric path followed in the quartz inclusions (sample cooled down from high temperatures) is different from the path followed in the other set of experiments (sample stretched from ambient temperature). This led us to hypothesize that cavitation in water might be path-dependent, but we ruled out this scenario by our acoustic measurements at high temperature [35]: they give cavitation for thermodynamic parameters at which some of the inclusions are still metastable, further cooling being necessary to make them cavitate.

We are therefore left with two explanations involving impurities: either destabilizing impurities present in all experiments except some of the quartz inclusions, which could thus reach the homogeneous cavitation limit; or stabilizing impurities present only in some quartz inclusions, which would allow them to survive conditions at which cavitation is observed in other experiments. We refer the reader to [35] for a more detailed discussion.

\section{Other liquids}

Because the results of acoustic cavitation in water are found to be so far from the CNT prediction, we decided to investigate other liquids to see if the discrepancy was specific to water [41]. Note that this study was performed with our former method to estimate the 
pressure, which is expected to give values less negative than the actual ones; see [2] nd [35] for details.

We have studied heavy water, which has nearly the same surface tension as water: we found the same cavitation pressure in both isotopes (see figure 6), so that the discrepancy with CNT is the same for normal and heavy water. We then turned to other liquids (ethanol and heptane) because they both have a similar surface tension, around three times smaller than water, and dimethylsulfoxide (DMSO) as an intermediate case. The results are displayed in figure 6 . The agreement with CNT is good for ethanol and heptane; the remaining discrepancy might be due in part to the underestimated amplitude of the negative pressure. For DMSO, CNT and experiment disagree, but to a lesser extent than for water. We therefore have the trend that the discrepancy between CNT and acoustic cavitation pressures increases for liquids with higher surface tensions. We plan to investigate this theoretically by treating cavitation in a more elaborate way than with CNT, e.g. using density functional theory.
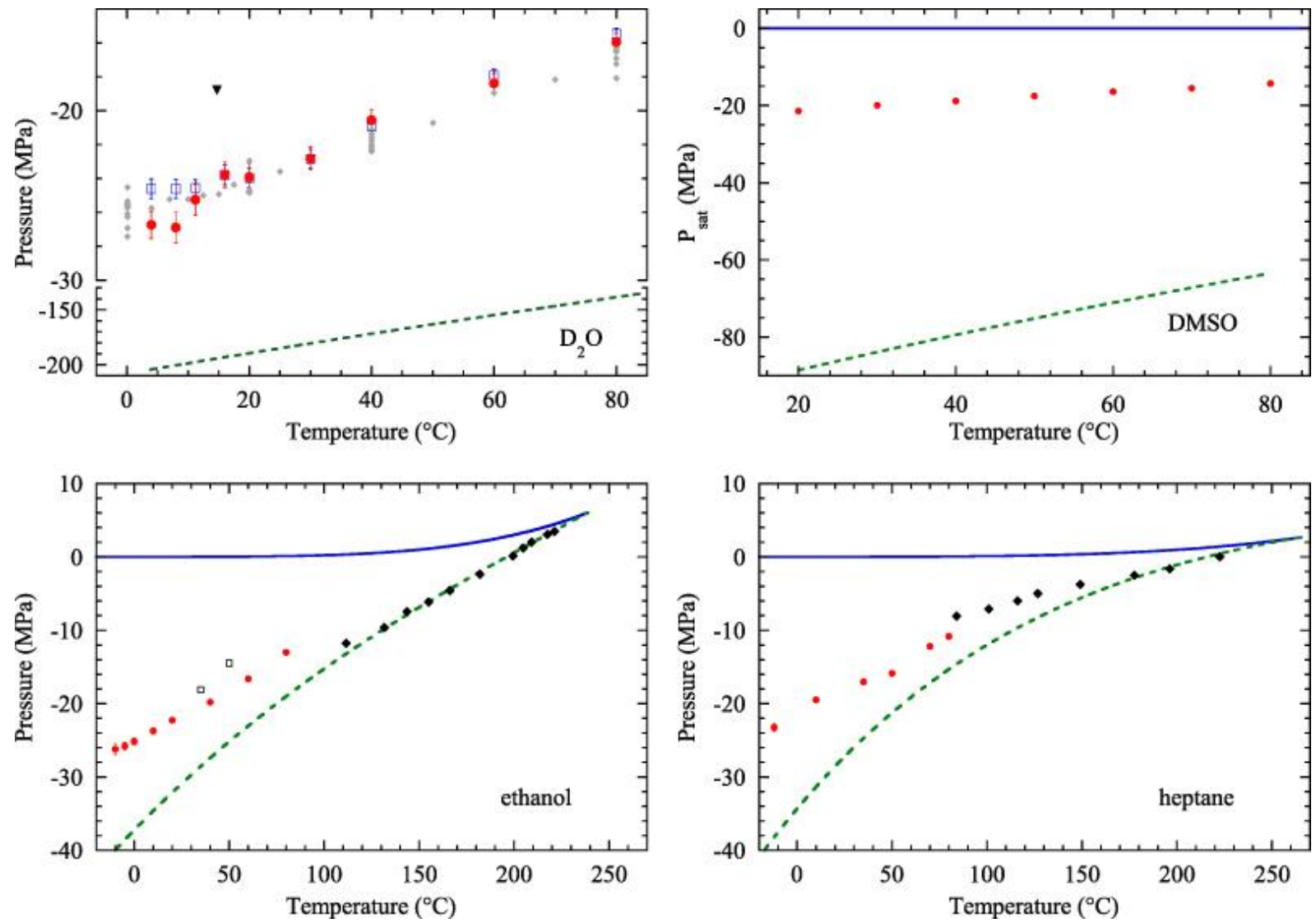

Figure 6. Cavitation pressure as a function of temperature for heavy water, DMSO, ethanol and heptane. Each panel displays the saturated vapour pressure (solid blue curve), $P_{\text {cav }}$ in the acoustic experiment (filled red circles) and the prediction of CNT, equation (6), with $\Gamma_{0} V \tau=10^{19}$ (dashed green curve). The results for heavy water (open blue and filled red circles) are close to those for normal water (grey diamonds). Experimental data from previous work is also included: [24] (filled black triangle), [42] (empty black squares) and [43] (filled black diamonds). Reprinted with permission from [41]. 


\section{Conclusion-perspectives}

Comparison of the different techniques to study stretched water reveals a surprising gap in the experimental data. Several techniques reach a cavitation pressure that is at most $-30 \mathrm{MPa}$, whereas a pressure down to $-140 \mathrm{MPa}$ is believed to have been reached in a quartz inclusion. It would be interesting to have an idea of the microscopic nucleation mechanism at play in the different systems. A promising direction is to use the nucleation theorem [44]. If accurate cavitation statistics can be measured, it allows us to derive the size of the critical bubble for nucleation. We have applied this analysis to the acoustic experiment and found that the critical bubble was equivalent to a hole in the liquid around $10 \mathrm{~nm}^{3}$ (see [35] for details). We plan to use the same approach in the case of quartz inclusions to see how different the critical bubble is.

Measuring the cavitation limit is not sufficient: we need also to measure the properties of the liquid in the metastable state. We have been able to measure the equation of state of water at room temperature down to $-26 \mathrm{MPa}$. This challenging experiment confirms the validity of the extrapolation of the IAPWS EoS. The question remains open for other temperatures and more negative pressure. To elucidate this issue, we will use the technique of quartz inclusions, because it is the only one able to reach large degrees of metastability, close to CNT predictions. We plan to perform extensive Brillouin scattering measurements on inclusions with different densities to map out the EoS.

We can think of other types of measurements, e.g. calorimetry. A possible direction to get the heat capacity of metastable water is to use new artificial trees, with a larger volume fraction of bulk water cavities inside the hydrogel. The study of other liquids, by experiment and also by more elaborated nucleation theories capturing the different microscopic interactions, is another exciting direction offered by the largely unexplored region of negative pressures.

\section{Acknowledgments}

We are indebted to C Austen Angell and Robin J Speedy for helpful discussions, for their continuous encouragements, and for giving us permission to reproduce figure 1 . This research has been funded by the ERC under the European Community's FP7 Grant Agreement 240113, by the Agence Nationale de la Recherche ANR Grant 09-BLAN-0404-01 and the Russian Fund of Basic Investigations Grant 10-05-00882. 


\section{References}

[1]Debenedetti P G 1996 Metastable liquids (Princeton, NJ: Princeton University Press)

[2]Herbert E, Balibar S and Caupin F 2006 Cavitation pressure in water Phys. Rev. E 74041603

[3]Tyree M T and Zimmermann M H 2002 Xylem Structure and the Ascent of Sap (Number XIV in Springer Series in Wood Science) 2nd edn (Berlin: Springer)

[4]Cochard H 2006 Cavitation in trees C. R. Phys. 7 1018-26

[5]Speedy R J and Angell C A 1976 Isothermal compressibility of supercooled water and evidence for a thermodynamic singularity at $-45^{\circ} \mathrm{C} \mathrm{J}$. Chem. Phys. $65851-8$

[6]Debenedetti P G 2003 Supercooled and glassy water J. Phys.: Condens. Matter 15 R1669-726

[7]Speedy R J 1982 Stability-limit conjecture. an interpretation of the properties of water J. Phys. Chem. 86 982-91

[8]Speedy R J 2004 Comment on 'Supercooled and glassy water' J. Phys.: Condens. Matter 16 6811-3

[9]Debenedetti P G 2004 Reply to comment on 'Supercooled and glassy water' J. Phys.: Condens. Matter 16 6815-7

[10]Caupin F, Balibar S and Maris H J 2001 Anomaly in the stability limit of liquid He-3 Phys. Rev. Lett. 87145302

[11]Poole P H, Sciortino F, Essmann U and Stanley HE 1992 Phase behaviour of metastable water Nature 360 324-8

[12]Hideki T 1996 Phase behaviors of supercooled water: reconciling a critical point of amorphous ices with spinodal instability J. Chem. Phys. 105 5099-111

[13]Brovchenko I, Geiger A and Oleinikova A 2005 Liquid-liquid phase transitions in supercooled water studied by computer simulations of various water models J. Chem. Phys. 123044515

[14]Brovchenko I and Oleinikova A 2008 Multiple phases of liquid water ChemPhysChem 9 2660-75

[15]F Sciortino, Poole P H, Essmann U and Stanley H E 1997 Line of compressibility maxima in the phase diagram of supercooled water Phys. Rev. E 55 727-37

[16]Liu Y, Panagiotopoulos A Z and Debenedetti P G 2009 Low-temperature fluid-phase behavior of ST2 water J. Chem. Phys. 131104508

[17]Meyer J 1911 Zur Kenntnis des negativen Druckes in Flüssigkeiten Abh. Dtsch. Bunsen-Ges. 6 1-53

[18]Berthelot M 1850 Sur quelques phénomènes de dilatation forcée des liquides Ann. Chim. Phys. 30 
[19]Briggs L J 1950 Limiting negative pressure of water J. Appl. Phys. 21 721-2

[20]Winnick J 1971 PVT behavior of water at negative pressures J. Chem. Phys. 552092

[21]Macdonald J R 1972 Reconsideration of an experiment on water under negative pressure J. Chem. Phys. 573793

[22]Huang HS 1973 PVT behavior of water at negative pressures: capillary tube deformation effects J. Chem. Phys. 596191

[23]Henderson S J and Speedy R J 1980 A Berthelot-Bourdon tube method for studying water under tension J. Phys. E: Sci. Instrum. 13778

[24]Henderson S J and Speedy $R J 1987$ Temperature of maximum density in water at negative pressure J. Phys. Chem. 91 3062-8

[25]Staudenraus J and Eisenmenger W 1993 Fiber-optic probe hydrophone for ultrasonic and shockwave measurements in water Ultrasonics 31 267-73

[26]Arvengas A, Davitt K and Caupin F 2011 Fiber optic probe hydrophone for the study of acoustic cavitation in water Rev. Sci. Instrum. 82034904

[27]Davitt K, Rolley E, Caupin F, Arvengas A and Balibar S 2010 Equation of state of water under negative pressure J. Chem. Phys. 133174507

[28]Wagner W 1999 The IAPWS formulation 1995 for the thermodynamic properties of ordinary water substance for general and scientific use J. Phys. Chem. Ref. Data 31387

[29]Revised release on the IAPWS formulation 1995 for the thermodynamic properties of ordinary water substance for general and scientific use 2009 International Association for the Properties of Water and Steam www.iapws.org/relguide/IAPWS95-Rev.pdf.

[30]Caupin F 2005 Liquid-vapor interface, cavitation, and the phase diagram of water Phys. Rev. E 71 051605

[31] Hiro K, Ohde $Y$ and Tanzawa $Y 2003$ Stagnations of increasing trends in negative pressure with repeated cavitation in water/metal Berthelot tubes as a result of mechanical sealing J. Phys. D: Appl. Phys. 36 592-7

[32] Wurster C, Köhler M, Pecha R, Eisenmenger W, Suhr D, Irmer U, Brümmer F and Hülser D 1995 Negative pressure measurements of water using the glass fiber optic hydrophone Proc. 1st World

Congr. on Ultrasonics (Berlin,) ed J Herbertz pp 635-8

[33] Galloway WJ 1954 An experimental study of acoustically induced cavitation in liquids J. Acoust. Soc. Am. 26849

[34]Greenspan M and Tschiegg C E 1967 Radiation-induced acoustic cavitation apparatus and some results J. Res. Natl Bur. Stand. C 71 299-312 
[35]Davitt K, Arvengas A and Caupin F 2010 Water at the cavitation limit: density of the metastable liquid and size of the critical bubble Eur. Phys. Lett. 9016002

[36] Wheeler T D and Stroock A D 2008 The transpiration of water at negative pressures in a synthetic tree Nature 455 208-12

[37]Caupin F and Herbert E 2006 Cavitation in water: a review C. R. Phys. 7 1000-17

[38]Zheng Q, Durben D J, Wolf $G H$ and Angell C A 1991 Liquids at large negative pressures: water at the homogeneous nucleation limit Science 254 829-32

[39]Alvarenga A D, Grimsditch M and Bodnar R J 1993 Elastic properties of water under negative pressures J. Chem. Phys. 98 8392-6

[40]Shmulovich K I, Mercury L, Thiéry R, Ramboz C and El Mekki M 2009 Experimental superheating of water and aqueous solutions Geochim. Cosmochim. Acta 73 2457-70

[41]Arvengas A, Herbert E, Cersoy S, Davitt K and Caupin F 2011 Cavitation in heavy water and other liquids J. Phys. Chem. B 115 14240-5

[42]Ohde $Y$, Watanabe $H$, Hiro K, Motoshita K and Tanzawa $Y 1993$ Raising of negative pressure to around-200 bar for some organic liquids in a metal berthelot tube J. Phys. D: Appl. Phys. 261188

[43]Vinogradov V E and Pavlov P A 2000 The boundary of limiting superheats ofn-heptane, ethanol, benzene, and toluene in the region of negative pressures High Temp. 38 379-83

[44] Oxtoby D W and Kashchiev D 1994 A general relation between the nucleation work and the size of the nucleus in multicomponent nucleation J. Chem. Phys. 100 7665-71 